\title{
Detection of an insertion deletion of region 8q13-q21.2 in a patient with Duane syndrome: implications for mapping and cloning a Duane gene
}

\author{
G Calabrese ${ }^{1}$ L Stuppia ${ }^{1}$, E M orizio ${ }^{1}$, P G uanciali Franchi $^{1}$, F Pompetti $^{1}$, \\ R Mingarelli ${ }^{2,3}, \mathrm{~T}$ M arsilio ${ }^{4}, \mathrm{M}$ R occhi ${ }^{5}, \mathrm{PE}$ Gallenga ${ }^{4}, \mathrm{G}$ Palka ${ }^{1}$ and B D'allapiccola ${ }^{2,3}$ \\ ${ }^{1} C$ attedra di $G$ enetica and ${ }^{4} C$ attedra di $O$ ftalmologia, U niversità degli Studi ' $G$ D 'A nnunzio', Chieti \\ ${ }^{2} \mathrm{M}$ endel-CSS I RCSS, Rome \\ ${ }^{3} \mathrm{C}$ attedra di G enetica M edica, U niversità Tor Vergata, Rome \\ ${ }^{5}$ I stituto di G enetica, Università di B ari, I taly
}

\begin{abstract}
Duane syndrome (MIM126800) is an autosomal dominant disease responsible for $1 \%$ of all strabismus cases and has been related to a 8q12-13 contiguous gene syndrome. We report on an insertion of chromosome region 8q13-q21.2 on to band $6 q 25$ in a patient presenting with Duane syndrome, mental retardation, and other dysmorphisms. FISH analysis using chromosome 8 radiation hybrid LIA2L indicated a concurrent deletion within the $8 q$ rearranged region. These results were corroborated by STR-PCR analysis and FISH using YAC contig WC8.8 disclosed a deletion in 8q13. Comparison of the two known patients with Duane syndrome associated with deletion of $8 q$ identifies a small region of overlap (SRO) of $<3 \mathrm{~cm}$ extending from D8S533 and D8S1767 in which a Duane syndrome locus is assigned. In addition YAC analysis in our patient showed that $8 q$ rearrangement was rather complex since $8 q$ deletion and insertion occurred in two distinct segments separated by a region which maintained its location on 8q.
\end{abstract}

Keywords: chromosome 8; deletion; Duane syndrome; mapping; complex rearrangement

\section{Introduction}

Duane syndrome is a primary form of strabismus consisting of an ocular motility defect with absent or severely limited abduction and variable limitation of

Correspondence: Giandomenico Palka, Via B Buozzi 93-65121 Pescara, Italy. Tel: + 39871355 4137; Fax: + 39871 355 4135; E-mail: gdpalka@unich.it.

R eceived 11 July 1997; revised 16 September 1997; accepted 17 November 1997 adduction, associated with bilateral globe retraction and narrowing palpebral fissure. ${ }^{1}$ This autosomal dominant (M IM 126800) disorder is responsible for approximately $1 \%$ of all strabismus cases. D uane syndrome has been clinically subclassified by $\mathrm{Huber}$ in three forms which cause deficiency of binocular sight due to altered ocular motility, eventually associated with nistagmus and other ocular anomalies. The pathogenesis of this syndrome is unknown but, in some patients at least, a muscular/neuronal origin has been proved. ${ }^{2-3}$ 
A lthough Duane syndrome is considered a clinically distinct condition, genetic heterogeneity has been suspected. In fact a few atypical cases of Duane syndrome have been described in association with deafness, renal defects, muscular, and skeletal anomalies $^{4-7}$ and chromosome imbalances. ${ }^{7-10}$ In particular a contiguous gene syndrome consisting of $D$ uane syndrome, branchio-oto-renal syndrome (BOR), hydrocephalus and trapeze aplasia was found in a patient with $8 q 12.2-q 21.2$ deletion. ${ }^{7}$ We report on an insertion deletion of chromosome 8q13-q21.2 in a patient presenting with Duane syndrome type I, severe mental retardation, and minor limbs abnormalities. M olecular studies were carried out in order to analyse and map this D uane syndrome containing region.

\section{Materials and Methods}

Patient

A 7-year-old girl, the first child of unrelated parents, was referred for cytogenetic analysis on the basis of severe mental retardation and dysmorphisms. Family history was unremarkable. A t birth her length was $48 \mathrm{~cm}$ and weight $3250 \mathrm{~g}$. A t 7 years of age, she was $119 \mathrm{~cm}$ in height (5th centile) and weighted $25 \mathrm{~kg}$ (65th centile). On clinical evaluation she presented with microcephaly, bilateral deficiency of ocular abduction, impairment of adduction, bilateral globe retraction and narrowing of interpalpebral fissure in adduction (D uane syndrome type I according to H uber's classification) ${ }^{2}$ prominent nasal root, anteverted nares, and micrognathia (Figure 1). E ars were normally implanted and no preauricular pit, tag and pinna malformation were observed. The patient also had short neck, brachydactyly and left club foot. M ental retardation was severe. Routine laboratory investigations,

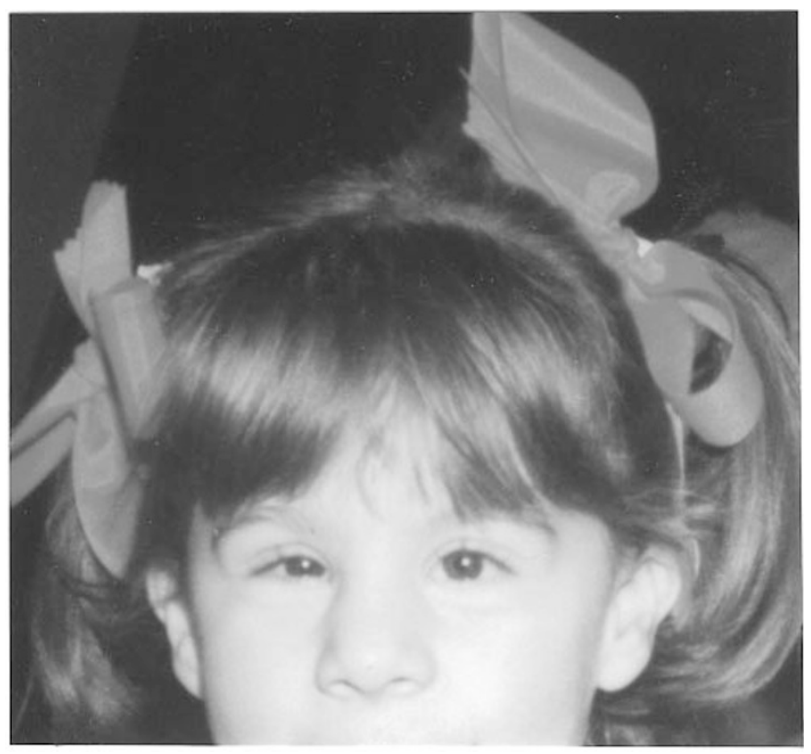

Figure 1 Proband phenotypes. skull X-ray, echocardiography, renal echography, and audiometry were normal. A n M R I revealed enlargement of lateral ventricle cornua.

\section{Cytogenetic Analysis and Fluorescence in situ \\ Hybridisation}

Chromosome studies were carried out on PHA -stimulated peripheral blood lymphocytes using GTG banding technique. $^{11}$

FISH analysis was performed according to Calabrese et al. ${ }^{12}$ Biotinylated and FITC-labelled chromosomes 6 and 8 painting probes (Cambio-Bouty, Milan, Italy), radiation hybrids LIA $2 \mathrm{~L}, \mathrm{HY}$ 95L and HY 95A 1 for region $8 \mathrm{q} 11-\mathrm{q} 21,{ }^{13}$ and YAC clones from contig W C8.8 (Whitehead Inst. M IT, $U S{ }^{14}$ ) were used. DNA from hybrids was A lu-PCR amplified with BK 33 primers. ${ }^{15} \mathrm{H}$ ybrid PCR products and YAC DNA were biotin and/or digoxigenin labelled by nick translation ( $L$ ife Technologies, G aithersburg MD, U SA ) prior to hybridisation.

\section{Genotyping}

Sixteen polymorphic sequence tagged sites (STS) mapping at 8q11.2-q21 were retrieved from the CEPH/G énéthon and Whitehead Institute databases ${ }^{16}$ and tested on DNA samples from the patient and her parents by PCR according to recommended protocols. PCR products were run on polyacrilamide or M etaphor XR (FMC, R ockland ME, USA) gels. A deletion in the patient was considered when three PCR experiments failed to amplify one parental allele.

\section{Results}

\section{Cytogenetic and FISH Analyses}

GTG banding in the proband revealed an insertion of segment $8 q 11.2 \rightarrow q 13$ on to $6 q 25$ (Figure 2), whilst the parents had normal karyotypes.

FISH experiments with chromosome 6 and 8 specific paintings confirmed chromosome $8 q$ insertion on to chromosome 6q25 (Figure 3a). In order to refine the

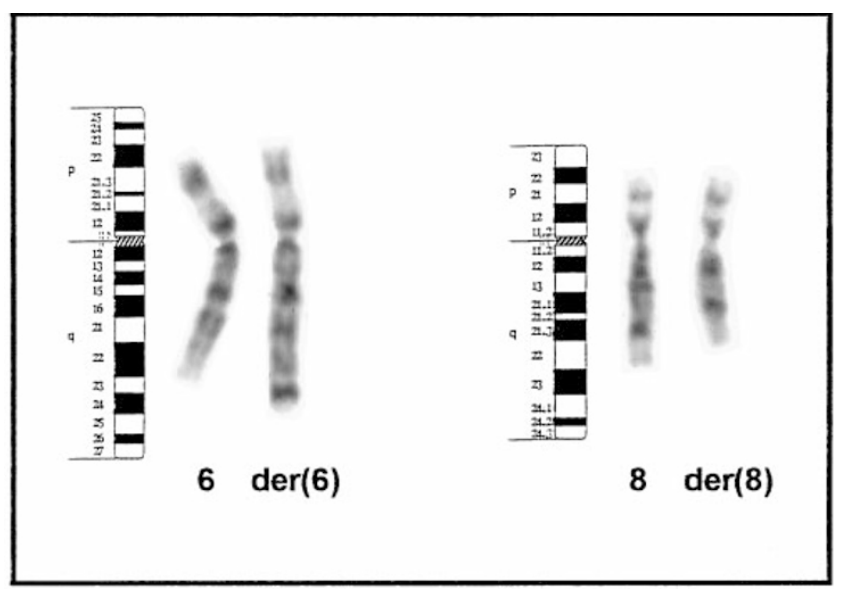

Figure 2 Partial karyotype of Duane patient showing an insertion of segment 8q11.2-q13 on to chromosome $6 q 25$. 


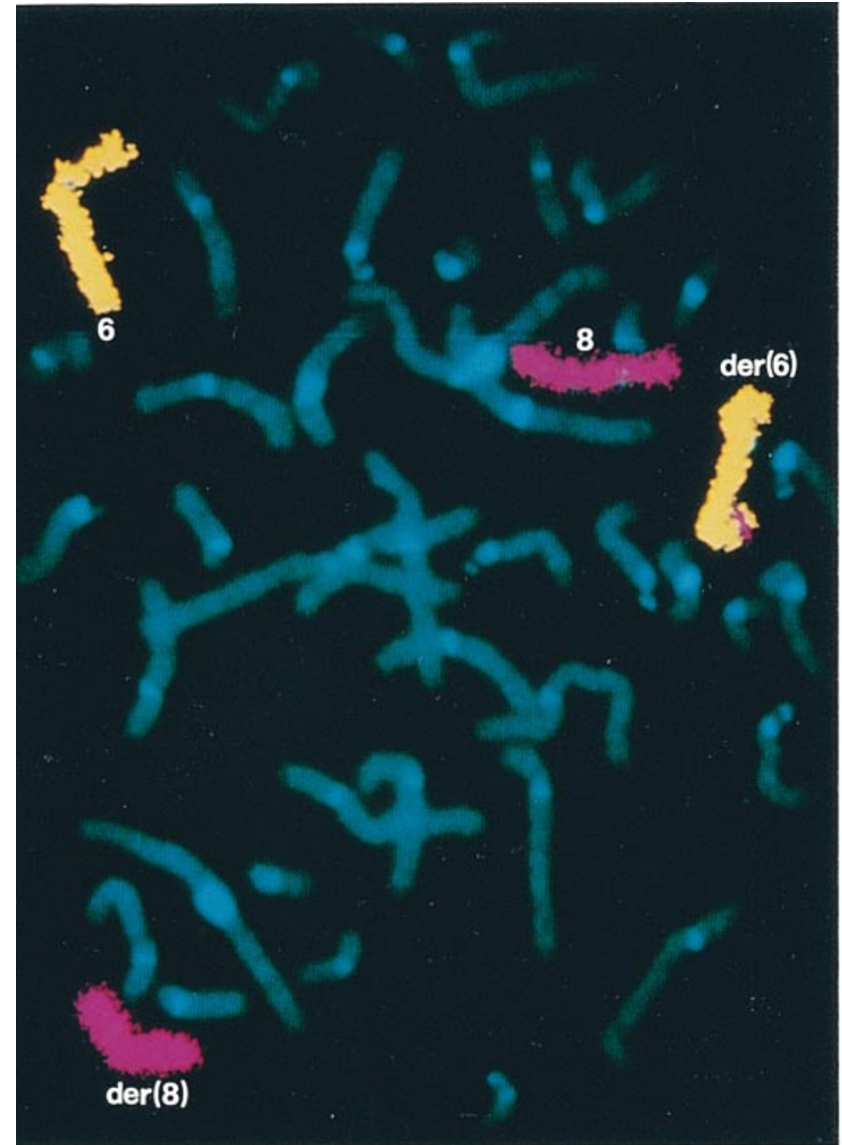

a

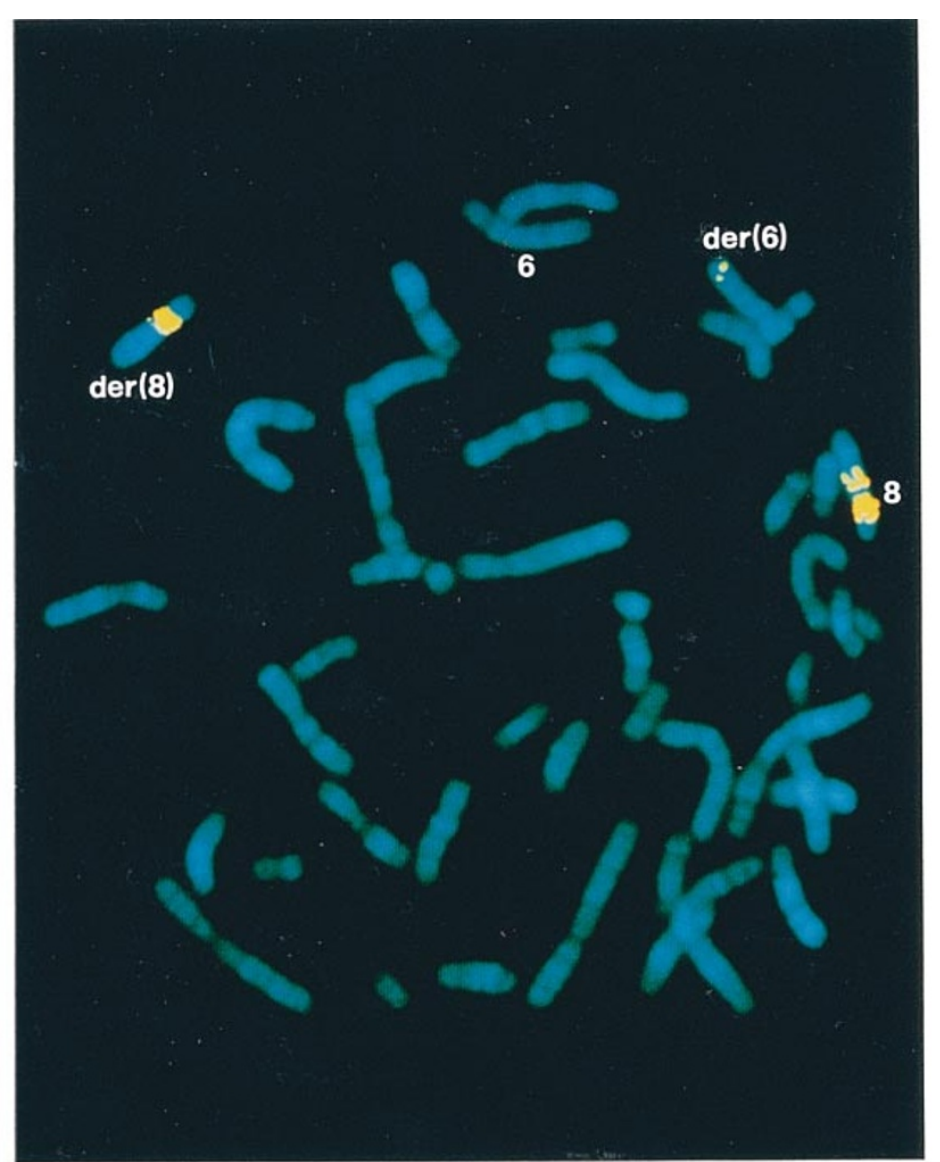

b

Figure 3 (a) FISH with painting probes for chromosome 6 (yellow signal) and chromosome 8 (red signal) showing insertion of material of chromosome 8 at band 6q25; (b) FISH with radiation hybrid LIA $2 \mathrm{~L}$. Chromosome $8 \mathrm{q}$ material on proximal q arm in $\operatorname{der}(8)$ and inserted in $\operatorname{der}(6)$ is fewer than that on normal chromosome 8 indicating that a deletion has occurred along with the insertion on to $6 q 25$.

chromosome 8 breakpoints, radiation hybrids LIA $2 \mathrm{~L}$, HY 95L and HY95A 1 were tested (Figure 4 and Figure 5). Hybrids HY 95L and HY95A 1 showed an apparent normal painting of $\operatorname{der}(8)$, while LIA $2 \mathrm{~L}$ was translocated onto $6 q 25$, a small signal being present onto der(6) (Figure $3 b$ ). These results relocated the $8 q$ breakpoints at $8 q 13$ and $8 q 21.2$ and suggested a concurrent deletion within this $8 q$ region.

\section{Genotyping}

M arkers D8S601, D8S1763, D8S510, D8S1841, D 8S1840, D 8S533, D 8S543, D 8S279, and D 8S286 were informative in this family (Figure 4). A nalysis of the polymorphic loci in the proband showed paternal allele loss of markers D 8S1841, D 8S1840 and D 8S533. These results were corroborated by STS analysis content of the three radiation hybrids. A s expected, only the translocated and deleted $\mathrm{LIA} 2 \mathrm{~L}$ hybrid was found positive for the deleted markers between D 8S1841 and D 8S533.

\section{Identification of YACS in the $8 q$ Rearranged Region}

Eighteen YAC clones were selected from the YAC contig WC 8.8 of the CEPH Mega-YAC Library based on the above STS/hybrid results. These clones were tested by FISH in the proband and in two unrelated controls (Table 1). A pparently all clones, except 850G 4, were not chimeric and disclosed a unique signal at 8q13-21.1 in the normal chromosome 8 in the patient. YAC clone $850 G 4$ showed signals at $8 q 13$ and $8 q 23$ (Figure 5a). FISH analysis using the most centromeric clones 799C11, 814A 6, 937E 2 and 751A 2 revealed signals in der(8), 937E 2 and 751A 2 disclosing a faint signal. Clones 942E 7 to $820 \mathrm{E} 6$ disclosed signals in normal chromosome 8, and no signal in $\operatorname{der}(8)$, while 


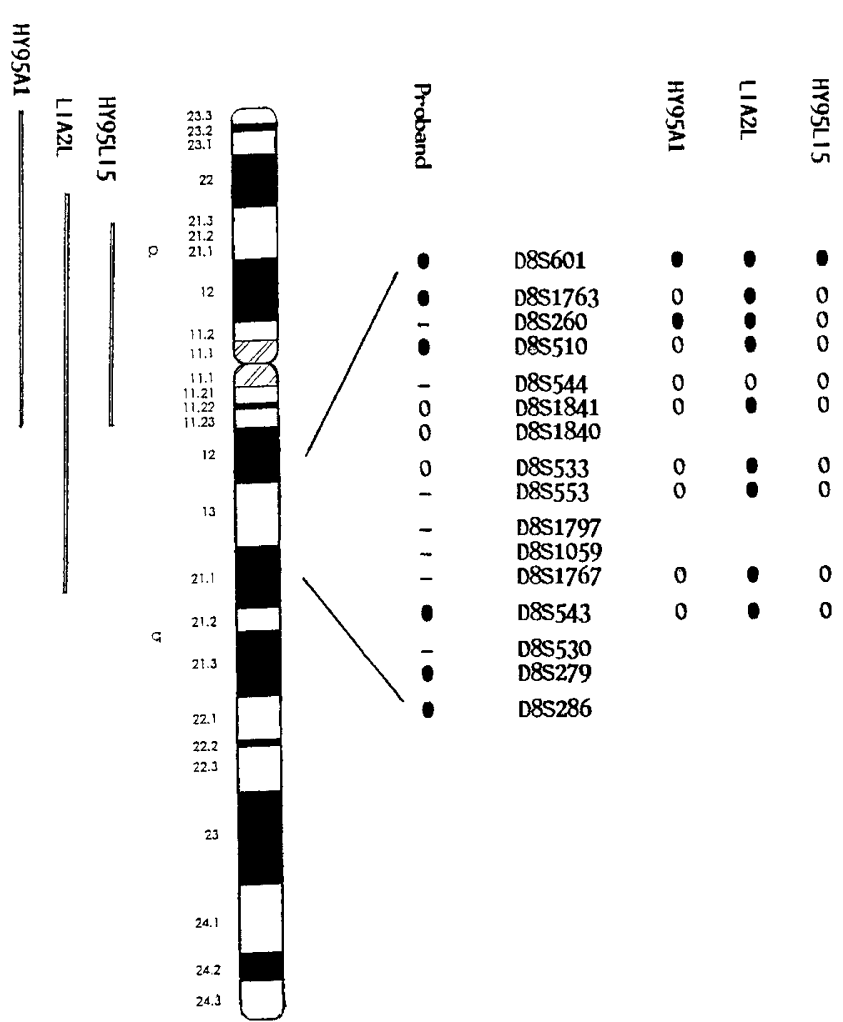

Figure 4 Radiation hybrids and polymorphic markers used for chromosome 8 analysis. From left to right, hybrids chromosome coverage, chromosome 8 diagram, and STS markers tested in the proband and hybrids are reported. E mpty circles indicate deleted markers. Uninformative STSs are shown with symbol (-).

910F5 showed signals in $\operatorname{der}(8)$ and $\operatorname{der}(6)$. These signals were small compared to the very large signal found in normal chromosome 8. FISH using the most telomeric clones 876D 10 to $944 \mathrm{C} 9$ displayed signals in normal chromosome 8 and der(6) (Table 1). Two-colour FISH experiments in interphase nuclei, combining clone 910F 5 with the more proximal overlapping clones 937E 2 or 751A 2, showed two separate large signals relative to the YAC loci on the normal chromosome 8. In addition there were one small signal for clone 910F 5 corresponding to the inserted region onto $\operatorname{der}(6)$, and two tiny signals, one for 910F 5 and one for the more proximal clones 937E 2 or 751A 2, which were always joined (Figure $5 b$ ). YAC clones were also tested in the proband's parents, which showed chromosome 8 hybridisation patterns fitting well with published mapping results.

A summary of STSs and FISH results in reported in Table 1.

\section{Discussion}

\section{Mapping a Locus for Duane Syndrome to $8 q 13$}

Vincent et $\mathrm{al}^{7}$ found a dir ins(8) (q24.11q13.3q21.13) which harboured a $10 \mathrm{~cm}$ deletion encompassing the $B O R$ syndrome related gene EYA 1 in a patient with $B O R$ syndrome associated with Duane syndrome, hydrocephalus, and trapeze aplasia. ${ }^{7,17}$ In the present study we have described a patient with $8 \mathrm{q}$ deletion associated with a complex phenotype, including $D$ uane syndrome, without any signs of BOR syndrome or other $8 q$ related disorders. ${ }^{7,17-19}$ Cytogenetic evidence of an insertion or region 8q13-21.2 on to chromosome $6 q 25$ was confirmed by FISH analysis using whole chromosome painting probes, radiation hybrids and YAC clones. In addition in situ hybridisation disclosed a deletion within band 8q13. Microsatellite analysis indicated that the deletion affected the paternal chromosome similar to the patient reported by $\mathrm{V}$ incent et al. $^{7}$ The deleted region boundaries were placed at D 8S510 and D8S543. Thus, the chromosomal loss spanned D 8S544 to D $8 S 1767$ in a region of about $6 \mathrm{~cm}$ according to published genetic distance. ${ }^{16}$ Comparison of the two patients with del $8 q$ associated with Duane syndrome pinpoints a small region of overlap (SRO) included between markers D 8S533 and D 8S1767. We have tentatively assigned a D uane syndrome locus to this region, which spans $3 \mathrm{~cm}$ and is located between the more proximal Friedreich ataxia with selective vitamin E deficiency locus ${ }^{19}$ and the more distal BOR syndrome locus. ${ }^{17,20}$

Finally, the association of Duane syndrome and hydrocephalus is very rare in the literature, whilst hydrocephalus and mental retardation are quite aspecific features of chromosome imbalances. ${ }^{21,22} \mathrm{Never-}$ theless, the concurrence of ventriculomegaly in the two patients with $D$ uane syndrome and del 8q supports the hypothesis of a contiguous gene syndrome at $8 q 13 .{ }^{7}$

\section{Rearrangement Characterisation}

FISH analysis of the $8 q$ region included between markers D 8S1763 and D 8S286 using Y A C clones of the contig WC8.8 disclosed a rather complex rearrangement in the proband. The deleted region spanned from YAC clone $942 E 7$ to $820 \mathrm{E} 6$, whilst the $8 \mathrm{q}$ region inserted on to chromosome $6 \mathrm{q} 25$ included the more distal clones 876D 10 to 944C 9 (Table 1). Clone 910F 5, mapping between 820E 6 and 876D 10, disclosed signals on to $6 \mathrm{q} 25$ and der(8). The concurrence of $910 \mathrm{~F} 5$ signals both on $\operatorname{der}(8)$ and $\operatorname{der}(6)$ suggests that the deletion 


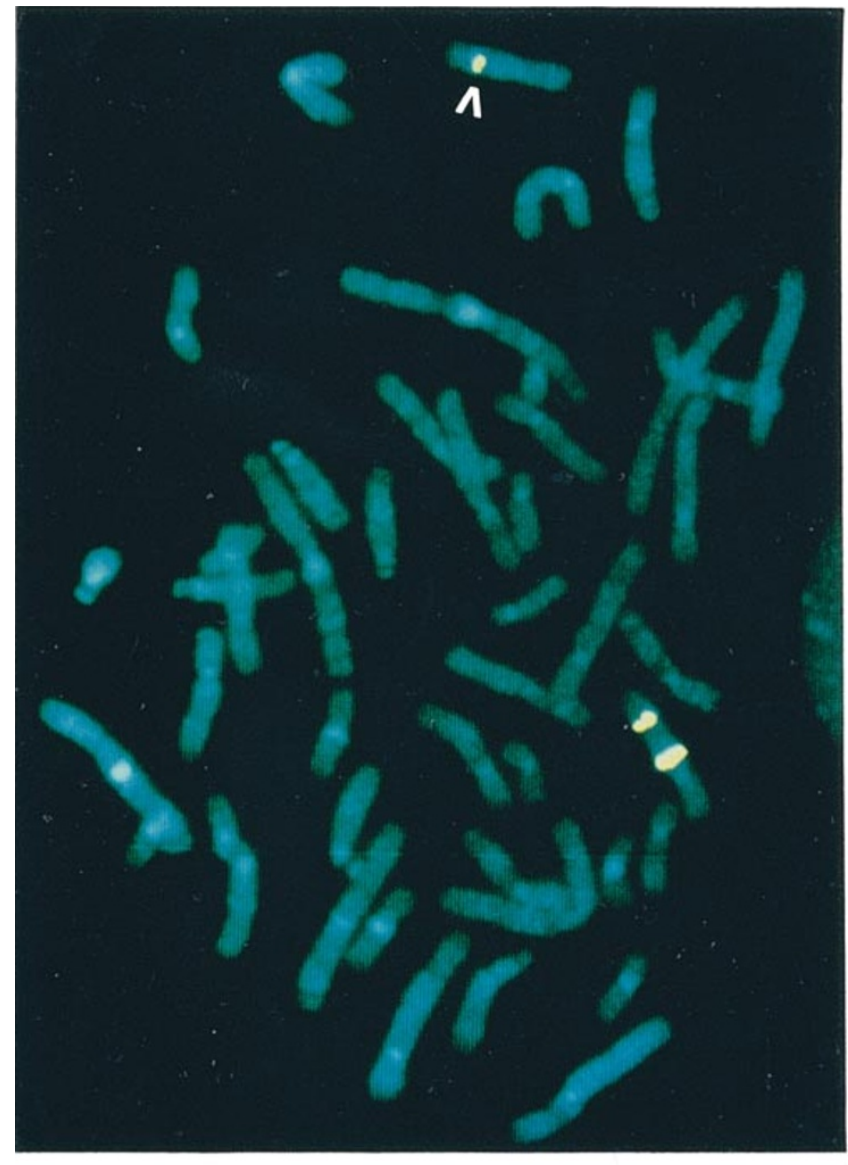

a

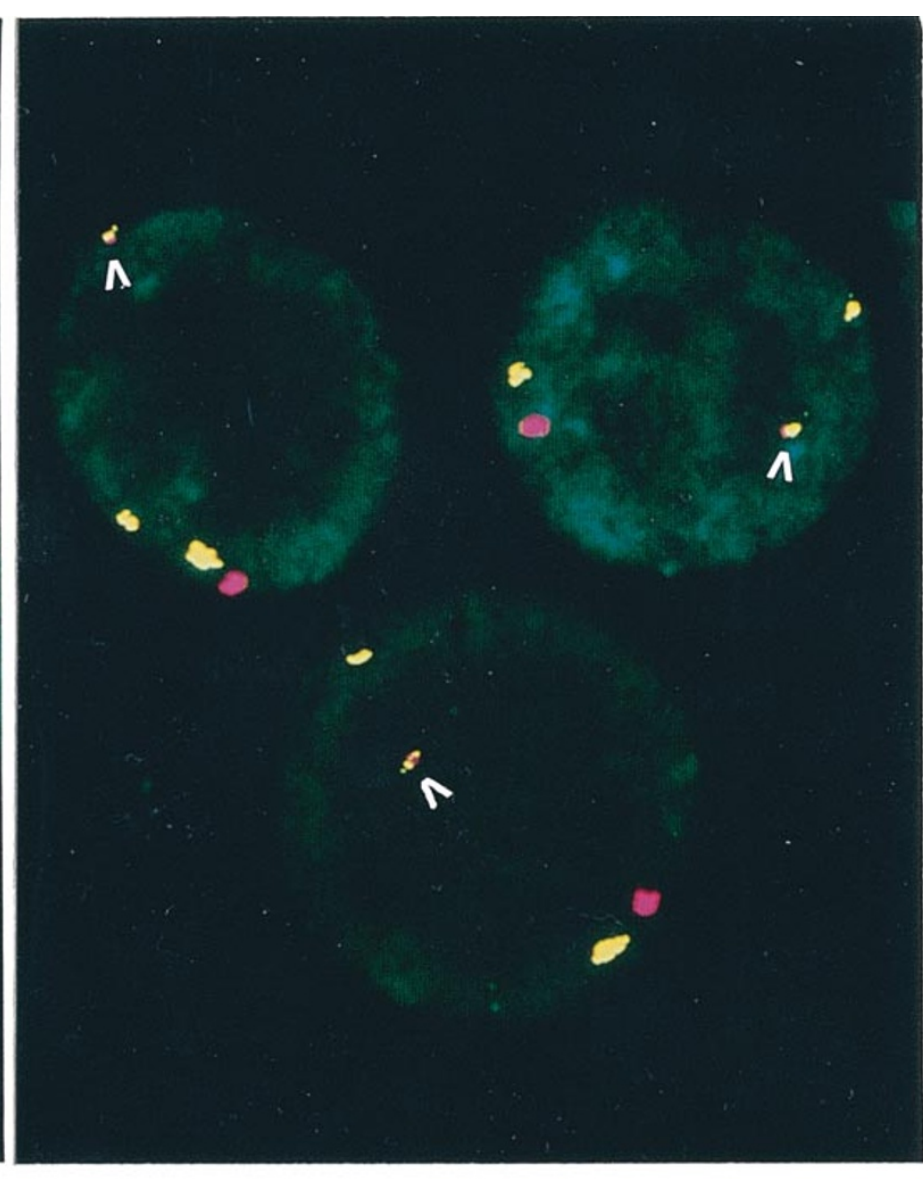

b

Figure 5 (a) FISH analysis with YAC clone 850G 4 showing signals on 8q13 and 8q23-24 on normal chromosome 8 and only the distal hybridization site on the $\operatorname{der}(8)$ (arrow) (b) two-color FISH analysis on interphase nuclei of the patient using clone 937E 2 (red signals) and clone 910F 5 (yellow signals). H ybridisation shows in each nucleus two large signals relative to the $\mathrm{AC}$ loci on the normal chromosome 8, one smaller yellow signal corresponding to the $8 q$ region inserted onto der(6), and two tiny signals, one red and one yellow, appearing joined (arrows).

and the insertion occurred on to two distinct segments separated by a region included in YAC 910F 5 which maintained its original location in 8q. Normal pattern of hybridisation using YACs in the parents indicated that the rearrangement in the patient was de novo and consisted of five breaks, including two each for deletion and insertion in $8 \mathrm{q}$, and one in $6 \mathrm{q} 25$.

FISH analysis using clone 910F 5 showed small signals both on der(8) and der(6) supporting a deletion also within this insert, which contains the distal limits of the deletion and the proximal breakpoint of the inserted region. This interpretation is in agreement with FISH experiments using clone 910F 5 and the more proximal overlapping clones 937E 2 or 751A 2, showing very small signals on $\operatorname{der}(8)$. FISH analysis of inter- phase nuclei, in addition to signals on normal chromosome 8 and der(6), also showed two tiny signals corresponding to $910 \mathrm{~F} 5$ and 937E 2 or 751A 2 (Figure $5 b)$. Joining of these signals indicated sequence fusion following deletion. These data also placed the proximal deletion boundary within the overlapping clones 937E 2 and 751A 2.

In conclusion, it is known that complex chromosome rearrangements could hamper a genotype-phenotype correlation. However, the association of D uane syndrome and deletion $8 q 13$ in the present patient and in another unrelated patient ${ }^{7}$ supports the location of a gene for D uane syndrome at $8 q 13$. The interval region around the putative $8 \mathrm{q} D$ uane gene has been narrowed down to a $<3 \mathrm{~cm}$ interval based on genetic-physical 
Table 1 STS and YAC content map from the $8 q$ region

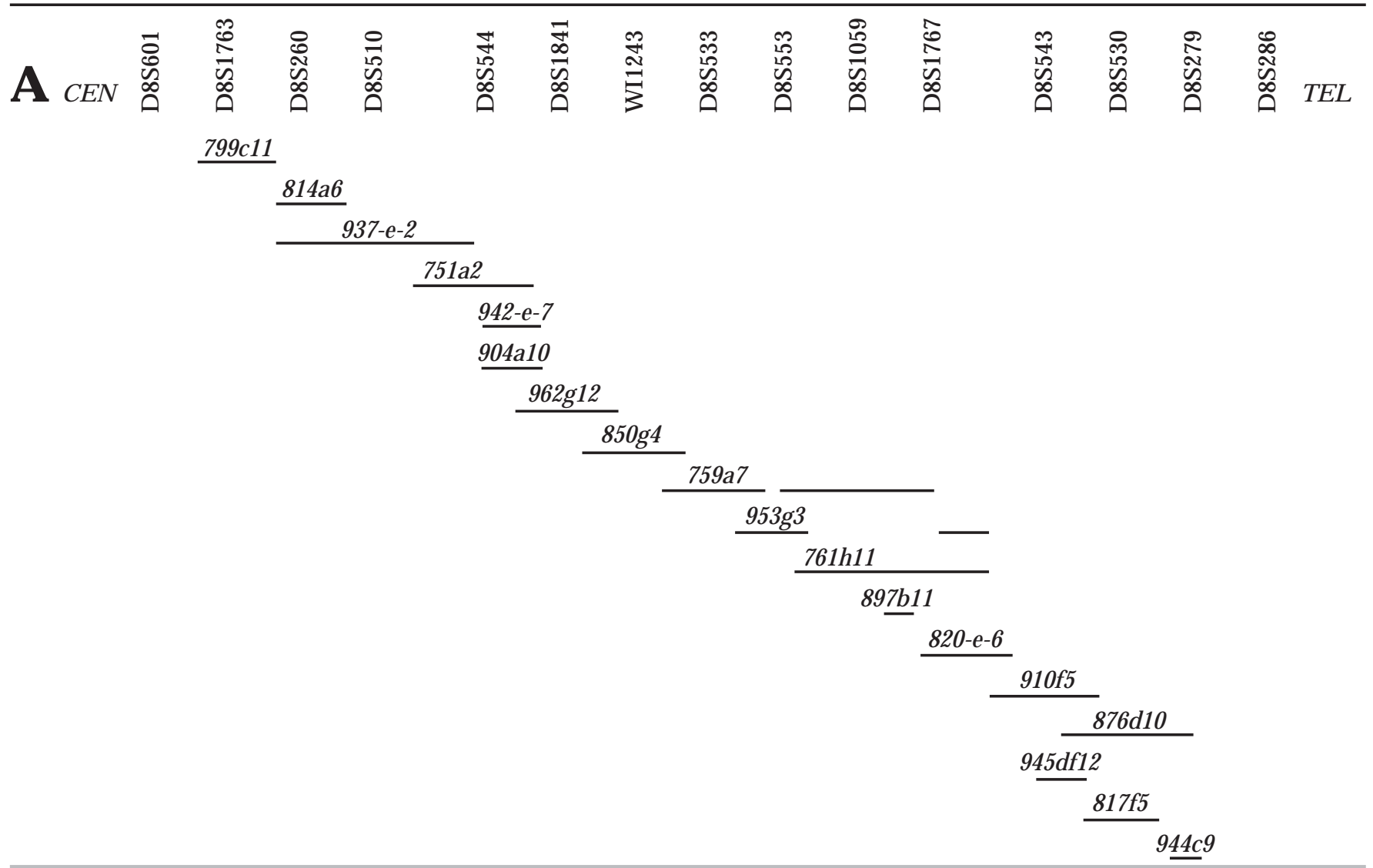

B

proband

Vincent et al, 1994

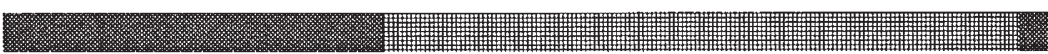

\begin{tabular}{|c|c|c|c|c|c|c|c|c|c|}
\hline & & & & & & Dua & n & 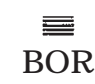 & \\
\hline $83 \mathrm{cM}$ & $84 \mathrm{cM}$ & $85 \mathrm{cM}$ & $87 \mathrm{cM}$ & $88 \mathrm{cM}$ & $88 \mathrm{cM}$ & $88 \mathrm{cM}$ & $90 \mathrm{cM}$ & $95 \mathrm{cM}$ & $98 \mathrm{cM}$ \\
\hline
\end{tabular}

Notes: (A) YAC contig of region $8 q 13-q 21$ (B) the deletion in the patient here reported, in Vincent's paper ${ }^{7}$ and the putative D uane locus interval are shown: dark bars for regions normally located on der(8), dashed bars for translocated segments, and gridded bars for deleted segments are reported in respect of YAC clone sites and/or STSs. Fredreich ataxia with vitamin $E$ deficiency (AVED) and BOR loci are also represented. The genetic distance between markers are as in the WC 8.8 contig map (W hitehead Institute, MIT, USA).

map of region ${ }^{23}$ included between D8S533 and D 8S1767.

\section{Acknowledgements}

The authors wish to thank C Sala at DIBIT Centre, Milan, for providing the YAC clones. The financial support of TelethonItaly (grant E 420) is gratefully acknowledged.

\section{References}

1 Bateman J B, I senberg SJ : G enetic aspects of strabismus. In: E mery A E H, R imoin D L (eds): Principles and Practice of Medical Genetics 2nd edn. Churchill Livingstone: E dinburgh, 1990, 726-727.

$2 \mathrm{H}$ uber A: E lectrophysiology of the retraction syndrome. B r J O phthalmol 1974; 58: 293-300.

3 Van Norden GK: Binocular Vision and Ocular Motility. CV M osby: St Louis, 1990, pp482-483. 
4 Pfaffenbach DD, Cross HE, Kearns TP: Congenital anomalies in D uane's retraction syndrome. A rch 0 phthalmol 1972; 88: 635-639.

5 Okihiro M M, Tasaki H, Nakano KK, Bennet BK: D uane syndrome and congenital upper limb anomalies: a familial occurrence. A rch Neurol 1977; 34: 174-179.

6 Ro A, Gummeson B, Orton RB, Cadera W: Duane's retraction syndrome. Can J Ophthalmol 1989 24: 200-203.

7 Vincent C, K alatzis V, Compain S et al: A proposed new contiguous gene syndrome on $8 \mathrm{q}$ consists of $\mathrm{B}$ ranchioO to-R enal (BOR) syndrome, D uane syndrome, a dominant form of hydrocephalus and trapeze aplasia: implications for the mapping of the BOR gene. $\mathrm{Hum} \mathrm{Mol} \mathrm{G} \mathrm{enet}$ 1994; 3: 1859-1866.

8 Cullen P, Rodgers CS, Callen DF et al: A ssociation of familial Duane anomaly and urogenital abnormalities with a bisatellited marker derived from chromosome 22. A m J M ed G enet 1993; 47: 925-930.

9 Chew CKS, Foster P, Hurst JA, Salmon JF: Duane's retraction syndrome associated with chromosome 4q27-31 segment deletion. A m J O phthalmol 1995; 119: 807-809.

10 Tibiletti M G, Sala E, Colombo D, A rlati S, Varisco T, La Placa G: Chromosome 22 marker in a child with Duane syndrome and urogenital abnormalities. A nn G énét 1996; 23: $234-236$

11 Seabright $M$ : A rapid banding technique for human chromosomes. L ancet 1971; ii: 971-972.

12 Calabrese G, Sallese M, Stornaiuolo A, Stuppia L, Palka $G$, De Blasi $A$ : Chromosome mapping of the human arrestin (SA G), $\beta$-arrestin 2 (ARRB2), and $\beta$-adrenergic receptor kinase 2 (A DR BK 2) genes. G enomics 1994; 23: 286-288.

13 A ntonacci $R$, Marzella $R$, Finelli $P$ et al: $A$ panel of subchromosomal painting libraries representing over 300 regions of the human genome. Cytogenet Cell G enet 1995; 68: $25-32$.

14 Chumak ov I M, R igault P, Le G all I et al. A Y A C contig of the human genome. Nature 1995; 377 (suppl): 175-299.

15 G ibbon B, Lillington D M , M onard S et al: Fluorescence in situ hybridization studies to characterise complete and partial monosomy 7 in myeloid disorders. G enes $C$ hromosom Cancer 1994; 10: 244-249.

16 D ib C, Fauré S, Fizames $C$ et al: A comprehensive genetic map of the human genome based on 5264 microsatellites. Nature 1996; 380: 152-154.

17 A bdelhak S, Kalatzis V, Heilig $\mathrm{R}$ et al: A human homologue of the $D$ rosophila eyes absent gene underlies Branchio-O to-R enal (BOR) syndrome and identifies a novel gene family. $N$ at $G$ enet 1997; 15: 157-164.

18 Schinzel A A, Robinson WP, Binkert F, Fanconi A : A n intertitial deletion of proximal $8 q(q 11-q 13)$ in a girl with Silver-R ussel syndrome-like features. Clin Dismorphol 1994; 3: 63-69.

19 Doerflinger $\mathrm{N}$, Linder $\mathrm{C}$, O uahchi $\mathrm{K}$ et al: A taxia with vitamin $E$ deficiency: refinement of genetic localization and analysis of linkage disequilibrium by using new markers in 14 families. Am J Hum Genet 1995; 56: 1116-1124.

20 K alatzis V, A bdelhak S, Compain S, Vincent C, Petit C: Characterization of a translocation-associated deletion defines the candidate region for the gene responsible for Branchio-O to-R enal syndrome. Genomics 1996; 34: 422- 425.

21 Prats J M, Garaizar C: D uane retraction syndrome associated with Chiari I malformation. Pediatr N eurol 1994; 10: 340.

22 On line Mendelian Inheritance in $\mathrm{M}$ an, OMIM (TM). Center for Medical Genetics, Johns Hopkins U niversity (Baltimore, MD) and $\mathrm{N}$ ational Center for Biotechnology Information, National Library of Medicine (Bethesda, MD ), 1996. World Wide Web URL:http://www.ncbi.nlm.nih.gov/O mim/.

23 Kumar S, Kimberling WJ, Lanyi A et al: Narrowing the genetic interval and yeast artificial chromosome map in the Branchio-Oto- $\mathrm{R}$ enal region on chromosome $8 \mathrm{q}$. G enomics 1996; 31: 71-79. 